\title{
Matricaria chamomilla L: propriedades farmacológicas
}

\author{
Matricaria chamomilla L: pharmacological properties \\ Matricaria chamomilla L: propiedades farmacológicas \\ Ana Raquel Ferreira da Costa SANTOS \\ José Henrique de Araújo CRUZ \\ Gymenna Maria Tenório GUÊNES \\ Abrahão Alves de OLIVEIRA FILHO \\ Maria Angélica Satyro Gomes ALVES \\ Unidade Acadêmica de Ciências Biológicas, Universidade Federal de Campina Grande (UFCG) 58700-970 Patos-PB, Brasil
}

\section{Resumo}

Introdução: A Matricaria chamomilla L., mais conhecida como camomila, vem sendo bastante utilizada na medicina popular devido suas consideráveis propriedades farmacológicas, como efeito anti-inflamatório, antioxidante, antimicrobiano e leve efeito sedativo. A camomila é muito utilizada sob a forma de infusões, com sabor agradável e aromático. Objetivo: Tendo em vista as várias finalidades e efeitos farmacológicos, objetivou-se neste trabalho realizar uma revisão de literatura acerca das propriedades farmacológicas da Matricaria chamomilla L. Metodologia: Essa busca foi realizada em artigos disponíveis nas bases de dados MEDLINE, Lilacs, PUBMED, BVS e monografias que atendiam aos requisitos do estudo em questão, no período de 2008 a 2018, revisando um total de 48 estudos. Discussão: Estudos demonstraram que a camomila é útil para tratamento da dor de estômago, síndrome do intestino irritável e insônia, além de possuir atividades bactericida e relaxante. A atividade antibacteriana dessa planta foi avaliada contra bactérias gram-negativas e os resultados comprovaram o efeito antibacteriano através dos principais componentes do óleo essencial, além dos flavonoides, ácidos fenólicos e ácidos graxos. Na Odontologia, sua efetividade é demonstrada através de sua ação benéfica contra gengivite, haja vista suas propriedades antimicrobianas e anti-inflamatórias. Conclusão: De acordo com a literatura, a Matricaria chamomilla L. apresenta efeitos antimicrobiano, antifúngico, antioxidante, anti-inflamatório e ansiolítico, os quais são explicados pelos componentes dos seus extratos, fazendo com que esta planta apresente grande importância clínica. Entretanto, mais estudos clínicos precisam ser realizados para enriquecer o conhecimento a respeito desta planta, de forma a ampliar o seu uso como medicamento fitoterápico.

Descritores: Fitoterapia; Plantas Medicinais; Farmacologia; Matricaria.

\begin{abstract}
Introduction: Matricaria chamomilla L., better known as chamomile, has been widely used in folk medicine because of its considerable pharmacological properties, such as anti-inflammatory, antioxidant, antimicrobial and mild sedative effects. Chamomile is widely used in the form of infusions, with a pleasant and aromatic flavor. Objective: The objective of this study was to carry out a literature review about the pharmacological properties of Matricaria chamomilla L. Methodology: This search was carried out in articles available in the MEDLINE, Lilacs, PUBMED, VHL and monographs that met the requirements of the study in question, from 2008 to 2018 , reviewing a total of 48 studies. Discussion: Studies have shown that chamomile is useful for treating stomach pain, irritable bowel syndrome and insomnia, and has bactericidal and relaxing activities. The antibacterial activity of this plant was evaluated against gram-negative bacteria and the results proved the antibacterial effect through the main components of the essential oil, besides the flavonoids, phenolic acids and fatty acids. In dentistry, its effectiveness is demonstrated through its beneficial action against gingivitis, given its antimicrobial and antiinflammatory properties. Conclusion: According to the literature, Matricaria chamomilla L. presents antimicrobial, antifungal, antioxidant, anti-inflammatory and anxiolytic effects, which are explained by the components of its extracts, making this plant of great clinical importance. However, more clinical studies need to be done to enrich the knowledge about this plant, in order to expand its use as a herbal medicine.
\end{abstract}

Descriptors: Phytotherapy; Plants, Medicinal; Pharmacology; Matricaria.

\section{Resumen}

Introducción: La Matricaria chamomilla L., más conocida como manzanilla, viene siendo bastante utilizada en la medicina popular debido a sus considerables propiedades farmacológicas, como efecto anti-inflamatorio, antioxidante, antimicrobiano y ligero efecto sedante. La camomila es muy utilizada en forma de infusiones, con sabor agradable y aromático. En el presente trabajo se analizaron los resultados obtenidos en el estudio de las propiedades farmacológicas de la Matricaria chamomilla L. Metodología: Esta búsqueda se realizó en artículos disponibles en las bases de datos MEDLINE, Lilacs, PUBMED, BVS y monografías que atendían a los requisitos del estudio en cuestión, en el período de 2008 a 2018, revisando un total de 48 estudios. Discusión: Estudios han demostrado que la manzanilla es útil para el tratamiento del dolor de estómago, síndrome del intestino irritable e insomnio, además de tener actividades bactericidas y relajantes. La actividad antibacteriana de esta planta fue evaluada contra bacterias gramnegativas y los resultados comprobaron el efecto antibacteriano a través de los principales componentes del aceite esencial, además de los flavonoides, ácidos fenólicos y ácidos grasos. En la Odontología, su efectividad es demostrada a través de su acción benéfica contra gingivitis, habida cuenta de sus propiedades antimicrobianas y antiinflamatorias. En la mayoría de los casos, la mayoría de las personas que sufren de esta enfermedad, se encuentran en la mayoría de los casos. Sin embargo, más estudios clínicos necesitan ser realizados para enriquecer el conocimiento acerca de esta planta, para ampliar su uso como medicamento fitoterápico.

Descriptores: Fitoterapia; Plantas Medicinales; Farmacología; Matricaria

\section{INTRODUÇÃO}

Os estudos sobre as plantas medicinais têm colaborado para propagar informações sobre sua importância terapêutica, reafirmando o conhecimento sobre elas acumulado há séculos. Todavia, os componentes químicos dessas plantas medicinais ainda não são totalmente conhecidos e precisam ser investigados ${ }^{1-4}$. Hodiernamente, a maioria das drogas são desenvolvidas a partir de constituintes de plantas medicinais, com base em suas aplicações ${ }^{5,6}$.

Matricaria chamomilla L. é uma espécie de planta medicinal bem conhecida da família Asteraceae, sendo nativa da Europa Meridional e Oriental $^{7}$. Esta planta é conhecida popularmente como camomila e é utilizada na medicina popular devido seus valores medicinais, consideráveis propriedades farmacológicas e também porque atestou um bom perfil de segurança ${ }^{8}$.

Embora a camomila seja usada amplamente em todo o mundo como um agente calmante, as pesquisas em humanos nesse campo ainda são muito 
limitadas. No único estudo controlado randomizado existente, pesquisadores constaram uma redução significativamente maior nos escores médios dos sintomas de ansiedade geral para camomila em comparação com placebo após 8 semanas de terapia ${ }^{9}$.

Essa planta medicinal é muito usada sob a forma de infusões ${ }^{10}$, possuindo sabor agradável e leve, com efeito sedativo e, além disso, numerosas pesquisas têm evidenciado que a camomila pode ser utilizada para vários fins, em virtude dos seus efeitos anti-inflamatórios ${ }^{11}, \quad$ antioxidante e antimicrobiano $^{12,13}$. Este último efeito farmacológico pode influenciar positivamente no controle do biofilme dentário, bem como as atividades antioxidante e anti-inflamatória têm papel protetor da cavidade bucal. Deste modo, a planta se mostra promissora para a pesquisa de novos tratamentos no âmbito da Odontologia.

Diante do exposto, objetivou-se neste trabalho realizar uma revisão de literatura acerca das propriedades farmacológicas da Matricaria chamomilla L.

\section{MATERIAL E MÉTODO}

O presente estudo trata-se de uma revisão bibliográfica narrativa, que analisa publicações amplas a respeito da planta Matricaria chamomilla $\mathrm{L}$. publicada em livros, artigos de revista impressas e/ou eletrônicas e monografias.

A pesquisa foi realizada no período de 20 de maio ao dia 21 de novembro de 2018. Como embasamento para a pesquisa, foram utilizados artigos científicos encontrados nas seguintes bases de dados: MEDLINE (Medical Literature Analysis and Retrieval Sistem Online), Lilacs (Centro LatinoAmericano e do Caribe de Informação em Ciências da Saúde), PUBMED, BVS (Biblioteca Virtual em Saúde), e monografias que atendiam aos requisitos do estudo em questão.

Os artigos utilizados como referências bibliográficas correspondem ao período entre 2008 a 2018, com exceção de artigos clássicos necessários para a escrita do trabalho. A pesquisa foi realizada tanto na língua nacional, Português, como em Inglês e Espanhol, revisando um total de 48 artigos. Foram utilizados como descritores isolados, ou combinados, para a busca em base de dados: "Matricaria Chamomilla L.", "Camomila na Odontologia", "Matricaria Chamomilla Anxiolytic", "Effects of Chamomile", "Chamomile and Dentistry", "Antifungical and Matricaria Chamomilla", "Antioxidant and Matricaria Chamomilla", "Camomila en la Odontología" para compor a base temática da revisão.

Importantes tópicos para discussão foram estudados e organizados da seguinte forma: a) aspectos botânicos; b) considerações bioquímicas e aplicações gerais; c) Atividade Antimicrobiana; d) Atividade Antioxidante; e) Atividade Antiinflamatória; f) Atividade Ansiolítica; g) Atividade Antifúngica.

\section{RESULTADOS E DISCUSSÃO}

- Aspectos botânicos

A Matricaria chamomilla $L$. é popularmente conhecida como, camomila italiana, camomila alemã, camomila selvagem ou camomila húngara ${ }^{14}$. A verdadeira camomila é uma planta anual com raízes finas e fusiformes que penetram o solo. A haste é ereta, fortemente ramificada e cresce a uma altura de 10 a $80 \mathrm{~cm}$. As folhas longas e estreitas são bipinadas e tripinadas. As cabeças das flores têm um diâmetro de 10 a $30 \mathrm{~mm}$ e são pedunculadas e heterogâmicas. As flores são tubulares amarelas e douradas com 5 dentes que possuem 1,5 a $2,5 \mathrm{~mm}$ de comprimento, terminando sempre em um tubo glanduloso. A planta possui entre 11 a 27 flores de cores brancas, as quais têm entre 6 a $11 \mathrm{~mm}$ de comprimento, $3,5 \mathrm{~mm}$ de largura e estão dispostas concentricamente. O receptáculo tem entre 6 a $8 \mathrm{~mm}$ de largura, com início plano e cônico e término em forma de cone oco - sendo este último uma característica distintiva muito importante da Matricaria chamomilla L. A fruta é um aquênio marrom amarelado ${ }^{7}$.

\section{- Propriedades fitoquímicas e farmacológicas}

A Matricaria chamomilla L. é uma das ervas medicinais mais conhecidas no Egito, Grécia e Roma $^{15}$. Compreende um grande grupo de interesse terapêutico e apresenta classes de compostos ativos como sesquiterpenos, flavonóides, cumarinas e poliacetilenos ${ }^{7}$. Possui onze compostos fenólicos bioativos, como a herniarina e umbeliferona (cumarina), ácido clorogênico e ácido cafeico (fenilpropanóides), apigenina, apigenina-7-Oglucosídeo, luteolina e luteolino-7-O-glicosídeo (flavonas), quercetina e rutina (flavonóis) e naringenina (flavanona) ${ }^{7}$.

A camomila é uma planta medicinal usada sob a forma de infusões, o que é apreciado em mais de um milhão de xícaras por dia ${ }^{10}$. Possui sabor agradável e aromático, bom efeito sedativo e uma diversidade de efeitos benéficos para a saúde que por muitas razões a faz ter uso universal. Numerosos estudos têm salientado que a camomila pode ser utilizada para vários fins, devido às suas atividades benéficas como anti-inflamatório além de sedativa ${ }^{11}$.

Relatos da literatura apontam que a camomila é útil ainda para tratamento da dor de estômago, síndrome do intestino irritável e insônia ${ }^{16,17,18}$, além de apresentar atividades bactericida, relaxante e acaricida. Em vários estudos em animais observaramse, efeitos ansiolíticos ${ }^{19}$, redução de colesterol e efeito antimutagênico, além de bom efeito sobre cicatrização de feridas $^{20}$ e propriedades antidiabéticas $^{21}$.

Em estudo de revisão da literatura realizado 
por Sharifi-Rad et al. ${ }^{18}$, observou-se que a camomila é uma planta medicinal reconhecida e cultivada em diversos países para fins comerciais com diversas finalidades, tais como: obtenção de sua essência azul, confecção de chá de ervas, e para usos farmacêuticos ou cosmecêuticos. O seu óleo essencial é obtido a partir das inflorescências frescas ou secas pela destilação a vapor. A composição volátil do óleo essencial, especialmente o conteúdo dos componentes valiosos $\alpha$-bisabolol e camazuleno, depende da parte da planta que for utilizada, origem e qualidade da fonte, fatores genéticos e ambientais. Além disso, outros parâmetros como a estação de colheita e métodos de extração, podem afetar o rendimento de extração dos óleos essenciais e extratos, sua composição e, portanto, sua bioatividade.

\section{- Atividade Antimicrobiana}

Muitas investigações asseguram o grande potencial antimicrobiano dos extratos vegetais. Os antimicrobianos oriundos dos metabólitos secundários são produzidos por muitas plantas como parte do processo normal do crescimento destas, bem como em resposta ao ataque de patógenos do meio ambiente $^{22-27}$.

$\mathrm{O}$ efeito antibacteriano das frações de camomila foi avaliado contra duas bactérias gramnegativas por Moricz et al. ${ }^{28}$, onde os resultados comprovaram seu efeito antibacteriano através de seus principais componentes do óleo essencial, incluindo cumarina, flavonóides, ácidos fenólicos e ácidos graxos.

Em uma pesquisa feita por Silva et al ${ }^{29}$ com objetivo de realizar uma análise fitoquímica e farmacológica do extrato bruto de Matricaria chamomilla $L$., foi observado que este foi eficiente contra cepas de S. Aureus. Em um estudo desenvolvido por Carvalho et al. ${ }^{30}$, com objetivo de avaliar a atividade antibacteriana do extrato etanólico de flores dessa planta, pôde-se concluir que este apresentou atividade antibacteriana frente à $P$. aeruginosa, porém não foi eficaz frente à $S$. aureus, E. coli e Salmonella enterica subsp. enterica sorovar Typhimurium.

Albuquerque et al. $^{31}$ avaliaram a atividade antimicrobiana in vitro do extrato da flor da camomila sobre microrganismos do biofilme dental. Os autores ainda compararam os resultados obtidos dos extratos hidroalcoólicos da planta $(0,84 \mathrm{~g} / \mathrm{mL})$ sobre as linhagens bacterianas, com os resultados obtidos com a Clorexidina $0,12 \%$. As bactérias testadas foram Streptococcus mutans, S. mitis, S. sanguinis, S. sobrinus e Lactobacillus casei. Esse estudo mostrou que o referido fitoterápico apresentou atividade antimicrobiana contra todas as linhagens de bactérias testadas, porém com menor efetividade que a clorexidina a $0,12 \%$.

- Atividade Antioxidante
Em um estudo realizado por AgatonovicKustrin et $\mathrm{al}^{32}$ quanto às suas atividades antioxidantes, verificou-se que os extratos das cabeças de flores e folhas da camomila são a fonte mais rica de atividade antioxidante, sendo o $\alpha$ bisabolol e o camazuleno os constituintes que possuem as maiores atividades antioxidantes.

Segundo Capuzzo et al. ${ }^{33}$ os óleos essenciais de Matricaria chamomilla L. contêm vários compostos bioativos, incluindo monoterpenos, sesquiterpenos, triterpenos e ácidos graxos. A hidrodestilação da camomila induz à formação de camazuleno. Este foi isolado do óleo essencial por cromatografia em coluna. A avaliação da capacidade antioxidante total do camazuleno confirmou uma boa atividade antioxidante comparada à do ácido ascórbico e $\alpha$-tocoferol. Os resultados deste trabalho mostraram que o camazuleno é um fator importante para o poder antioxidante do óleo essencial da camomila.

Jabri et al. $^{34}$, em um estudo pré-clínico, verificaram os efeitos protetores de extratos de camomila contra espécies reativas de oxigênio. Os resultados indicaram que o extrato inibe a produção das espécies reativas de oxigênio em neutrófilos e protege contra alterações hematológicas induzidas pelo etanol e estresse oxidativo dos eritrócitos. A hematoproteção oferecida pela camomila pode envolver, em parte, suas propriedades antioxidantes, bem como seu efeito sobre alguns mediadores intracelulares, como $\mathrm{H}_{2} \mathrm{O}_{2}$, ferro livre e cálcio.

Em um estudo in vitro focado em outro aspecto da atividade biológica de conjugados polifenólico-polissacarídico de camomila, suas propriedades antioxidantes na proteção de componentes do plasma sanguíneo contra o estresse oxidativo in vitro, Kolodziejczyk-Czepas et al. ${ }^{35}$ obtiveram resultados que indicam que os conjugados polifenólicos-polissacarídeos isolados de substâncias de $M$. chamomilla possuem propriedades antioxidantes. Os glicoconjugados macromoleculares de $M$. chamomilla podem ser úteis na criação de novos medicamentos ou suplementos dietéticos baseados na natureza, úteis na prevenção e tratamento de distúrbios mediados pelo estresse oxidativo.

Pereira et al. ${ }^{36}$ realizaram um trabalho que teve por com objetivo estudar e otimizar o processo de maceração dinâmica de Matricaria chamomilla L., de forma a obter extratos de inflorescência com maior teor de flavonóides e melhor atividade antioxidante. Usando uma abordagem multivariada, encontraram resultados que mostraram que com este modelo foram capazes de prever as propriedades do extrato. Além disso, o processo de extração desenvolvido mostrou-se confiável, eficiente e possível de ser realizado em escala para inflorescências de Matricaria chamomilla L. O 
extrato apresentou maior teor de flavonóides, apigenina e apigenina-7-glicosídeo além de uma alta atividade antioxidante.

\section{- Atividade Antiinflamatória}

Estudos realizados por Miguel et al. ${ }^{37}$ confirmaram o efeito anti-inflamatório do flavonóide apigenina, a qual é encontrada principalmente em sua forma glicosilada, a apigenina-7-glicosídeo na Matricaria chamomilla L. A atividade antiinflamatória foi confirmada por uma produção reduzida de TNF- $\alpha$ (fator de necrose tumoral $\alpha$ ) observada em camundongos tratados com apigenina após o tratamento com lipopolissacarideo (LPS).

Drummond et al. ${ }^{38}$ demonstraram em estudo clínico os efeitos benéficos da Matricaria chamomilla $L$. na inflamação sistêmica articular, na qual a função articular mecânica melhorou, bem como a dor no joelho e na parte inferior das costas foram atenuadas.

Quanto à cavidade bucal, em uma pesquisa realizada por Batista et al. ${ }^{39}$, com intuito de avaliar a efetividade dos extratos de camomila como enxaguatório bucal, concluiu-se que as lavagens bucais com o extrato da planta foram benéficas, pois possuiam propriedades antimicrobianas e antiinflamatórias. Estes efeitos são benéficos contra gengivite, por exemplo.

Em uma pesquisa pré-clínica feita por Rocha et al. $^{40}$, o (-) - $\alpha$-bisabolol foi testado em modelos padronizados de roedores por administração por gavagem nas doses de 100 e $200 \mathrm{mg} / \mathrm{kg}$ nos modelos de inflamação e 25 e $50 \mathrm{mg} / \mathrm{kg}$ nos modelos de nocicepção. Nos modelos inflamatórios de edema de pata induzido por carragenina e dextrano, os ratos tratados com (-) - $\alpha$-bisabolol apresentaram edemas menores em comparação aos animais tratados com o veículo. $O(-)$ - $\alpha$-bisabolol foi capaz de reduzir edemas de pata induzidos por 5-HT (5hidróxitriptamina), mas não edemas induzidos por histamina. O (-) - $\alpha$-bisabolol demonstrou atividade antinoceptiva nos modelos de nocicepção visceral induzida pelo ácido acético e na segunda fase do teste de nocicepção induzido pela administração intraplantar de formalina. $\mathrm{O}(-)$ - $\alpha$-bisabolol não teve nenhum efeito em um modelo de nocicepção térmica usando uma placa quente, mas foi capaz de diminuir a hipernocicepção inflamatória mecânica evocada pela carragenina. Esses achados sugerem que a ação antinociceptiva do (-) - $\alpha$-bisabolol não está ligada a um mecanismo central, mas está relacionada ao processo inflamatório. O (-) - $\alpha$-bisabolol foi capaz de diminuir a migração de leucócitos, extravasamentos de proteínas e a quantidade de TNF$\alpha$ para a cavidade peritoneal em resposta à carragenina. Adicionalmente, o (-) - $\alpha$-bisabolol reduziu a desgranulação de neutrófilos em resposta ao acetato de mirbol-miristato. Demonstramos, pela primeira vez, as atividades periféricas antiinflamatórias e antinociceptivas do (-) - $\alpha$ bisabolol.

Um estudo feito por Goes et al. ${ }^{41}$ avaliou a eficácia de um enxaguatório bucal contendo $1 \%$ do extrato de Matricaria Chamomilla L. na redução da gengivite e formação de biofilme em pacientes submetidos a tratamento ortodôntico com aparelhos fixos. Como resultado, o extrato reduziu o acúmulo de biofilme e sangramento gengival em pacientes com gengivite, provavelmente devido ao seu efeito antimicrobiano e anti-inflamatório.

- Atividade Ansiolítica

Evidências disponíveis sugerem a utilidade de alguns medicamentos fitoterápicos no alívio da ansiedade e depressão, mas dados conclusivos para mostrar à comunidade científica em relação os benefícios/riscos destes produtos em comparação aos produtos farmacêuticos atuais ainda são escassos ${ }^{42}$.

Sobre as propriedades ansiolíticas da Matricaria chamomilla L., testes clínicos realizados por Chang e $\mathrm{Chen}^{43}$ analisaram a eficiência da infusão da planta na depressão, insônia e tédio, bem como ansiedade em mulheres pós-parto. Foi verificado que a infusão atenuou a depressão e problemas de sono nessas mulheres.

Concordando com os dados do estudo citado acima, Amsterdan et al. ${ }^{19}$ examinou a ação antidepressiva do extrato de Matricaria chamomilla $L$. administrado por oito semanas por via oral em indivíduos com sintomas de ansiedade e depressão mórbida. $\mathrm{O}$ grupo de tratado com extrato dessa planta exibiu uma maior redução na média de pontuações na Avaliação de Depressão de Hamilton (HAM-A) e não apresentou efeitos colaterais significativos.

Uma pesquisa realizada por Mao et al. ${ }^{44}$, analisou o efeito do uso pronlongado da Matricaria chamomilla $L$. na prevenção do Transtorno da Ansiedade Generalizada e sua recaída. Como resultado, o tratamento reduziu significativamente o transtorno da ansiedade generalizada, que era classificada como sintomas moderados a graves, mas não reduziu significativamente a taxa de recaída da ansiedade.

Zick et al. $^{45}$ examinaram a eficácia e segurança da Matricaria chamomilla L. para o sono em pacientes com insônia, além de investigar os sintomas diurnos. No total, foram avaliados 34 pacientes. Os pesquisadores demonstraram que a o tratamento com a planta provocou efeitos de baixa a moderada intensidade na melhora na latência do sono, no despertar noturno e nos escores de gravidade de fadiga, mas nenhum efeito benéfico significativo foi observado no total de tempo de sono e na sua eficiência do sono.

Como visto no estudo realizado por Choi et al. ${ }^{46}$ novas pesquisas na área são necessárias para verificar de fato a influência que a planta tem para o controle da ansiedade, além de outros transtornos, 
para que possa ser bem aplicada e inserida no meio odontológico como coadjuvante na diminuição da ansiedade e medo do dentista.

\section{- Atividade Antifúngica}

Em se tratando dos efeitos antifúngicos da Matricaria chamomilla $L$., um estudo realizado por Tolouee et al. ${ }^{47}$ avaliou a atividade antifúngica do óleo essencial da flor da planta contra Aspergillus niger, dando-se ênfase no seu mecanismo de ação. Utilizando microscopia eletrônica, observou-se que o crescimento de A. niger foi inibido, com um máximo de $92,50 \%$ na mais alta concentração do óleo (1000 $\mu \mathrm{g} / \mathrm{mL}$ ). Um retardo acentuado na produção de conídios pelo fungo foi notado em relação à inibição do crescimento das hifas. Alterações morfológicas se devem ao efeito sobre a permeabilidade celular, através da interação direta do óleo essencial de Matricaria chamomilla L. com a membrana plasmática fúngica. Estes achados indicam o potencial do óleo essencial desta planta na prevenção da contaminação fúngica.

No estudo proposto por Jamalian et al. ${ }^{48}$ objetivando avaliar o efeito do óleo essencial de flores de Matricaria Chamomilla L., foi avaliado o seu efeito sobre dermatófitos e saprófitas oportunistas usando microbioensaio técnico. Os resultados deste estudo indicaram que esse óleo essencial pode ser considerado como um candidato potencial para compor formulações antifúngicas eficazes adequadas para o tratamento de dermatofitose e outras infecções fúngicas.

\section{CONCLUSÃO}

De acordo com a literatura estudada, observaram-se importantes atividades farmacológicas pré-clínicas e clínicas da Matricaria chamomilla L., dentre as quais pode-se citar atividade antimicrobiana, antioxidante, anti-inflamatória, ansiolítica e antifúngica. Essas atividades foram estudadas a partir de extratos, infusões e de testes com seus constituintes químicos. Desta forma, a Matricaria chamomilla $L$. apresenta-se promissora para diferentes tratamentos farmacológicos. Apesar disso, mais estudos clínicos precisam ser realizados para enriquecer o conhecimento atual a respeito desta planta.

\section{REFERÊNCIAS}

1. Severino VGP, Felixa MA, Silva MFGF, Lucarini R, Martins CHG. Chemical study of Hortia superba (Rutaceae) and investigation of the antimycobacterial activity of crude extracts and constituents isolated from Hortia species. Quím Nova. 2015;38(1):42-5.

2. Bardaji DK, Reis EB, Medeiros TC, Lucarini R, Crotti AE, Martins CH. Antibacterial activity of commercially available plant-derived essential oils against oral pathogenic bacteria. Nat Prod Res. 2016;30(10):1178-81.
3. Newman DJ, Cragg GM. Natural products as sources of new drugs from 1981 to 2014. J Nat Prod. 2016;79(3):629-61.

4. Suleimen E, Ibataev $\mathrm{ZH}$, Iskakova $\mathrm{ZH}$, Ishmuratova $\mathrm{M}$, Ross $\mathrm{S}$, Martins CHG. Constituent composition and biological activity of essential oil from Artemisia terrae-albae. Chem Nat Compd. 2016;52:173-75.

5. Jardak M, Elloumi-Mseddi J, Aifa S, Mnif S. Chemical composition, anti-biofilm activity and potential cytotoxic effect on cancer cells of Rosmarinus officinalis L. essential oil from Tunisia. Lipids Health Dis. 2017;16(1):190.

6. Habtemariam S. The therapeutic potential of rosemary (Rosmarinus officinalis) diterpenes for Alzheimer's disease. Evid Based Complement. Alternat Med. 2016;2016:2680409.

7. Singh O, Khanam Z, Misra N, Srivastava M. Chamomile (Matricaria chamomilla L.): An overview. Pharmacogn Rev. 2011;5(9):82-95.

8. Keefe JR, Mao JJ, Soeller I, Li QS, Amsterdam JD. Short-term open-label Chamomile (Matricaria chamomilla L.) therapy of moderate to severe generalized anxiety disorder. Phytomedicine. 2016;23(14):1699-705.

9. Amsterdam JD, Li Y, Soeller I, Rockwell K, Mao JJ, Shults J. A randomized, double-blind, placebocontrolled trial of oral Matricaria recutita (chamomile) extract therapy for generalized anxiety disorder. J Clin Psychopharmacol. 2009; 29(4):378-82.

10. Srivastava JK, Gupta S. Health promoting benefits of chamomile in the elderly population. In: Watson RR (Ed). Complementary and alternative therapies and the aging population an evidencebased approach. San Diego, USA: Academic Press Inc; 2010.

11. Srivastava JK, Shankar E, Gupta S. Chamomile: A herbal medicine of the past with bright future. Mol Med Rep.2010;3(6):895-901.

12. Braga FTMM, Santos ACF, Bueno PCP, Silveira RCCP, Santos CB, Bastos JK et al. Use of Chamomilla recutita in the prevention and treatment of oral mucositis in patients undergoing hematopoietic stem cell transplantation. Cancer Nurs. 2015;38(4):322-29.

13. Curra M, Martins MAT, Lauxen IS, Pellicioli ACA, Sant'Ana Filho M, Pavesi VCS et al. Efect of topical chamomile on immunohistochemical levels of IL-1 $\beta$ and TNF- $\alpha$ in 5-fuorouracilinduced oral mucositis in hamsters. Cancer Chemother Pharmacol. 2013;71(2):293-99.

14. Mekonnen A, Yitayew B, Tesema A, Taddese S. In vitro antimicrobial activity of essential oil of Thymus schimperi, Matricaria chamomilla, Eucalyptus globulus, and Rosmarinus officinalis. Int J Microbiol. 2016;2016:9545693.

15. Najla OA, Olfat AK, Kholoud SR , Enas ND, 
Hanan S. Hypoglycemic and Biochemical Effects of Matricaria Chamomilla Leave Extract in Streptozotocin-Induced Diabetic Rats. J Health Sci. 2012;2(5):43-8.

16.Zargaran A, Borhani-Haghighi A, Faridi P, Daneshamouz S, Kordafshari G, Mohagheghzadeh A. Potential effect and mechanism of action of topical chamomile (Matricaria chammomila L.) oil on migraine headache: a medical hypothesis. Med Hypotheses. 2014;83(5):566-69.

17. Srivastava JK, Pandey M, Gupta S. Chamomile, a novel and selective COX-2 inhibitor with antiinflammatory activity. Life Sci. 2009;85(19-20): 663-69.

18. Sharifi-Rad M, Nazaruk J, Polito L, Morais-Braga MFB, Rocha JE, Coutinho HDM et al. Matricariagenus as a source of antimicrobial agents: From farm to pharmacy and food applications. Microbiol Res. 2018;215:76-88.

19. Amsterdan JD, Shults J, Soeller I, Mao JJ, Rockwell K, Newberg AB. Chamomile (Matricaria recutita) may provide antidepressant activity in anxious, depressed humans: an exploratory study. Altern Ther Health Med. 2012;18(5):44-9.

20. Hashempur MH, Lari ZN, Ghoreishi PS, Daneshfard B, Ghasemi MS, Homayouni K, et al. A pilot randomized double-blind placebocontrolled trial on topical chamomile (Matricaria chamomilla L.) oil for severe carpal tunnel syndrome. Complement Ther Clin Pract. 2015;21(4):223-28.

21. Jarrahi M, Vafaei AA, Taherian AA, Miladi H, Rashidi Pour A. Evaluation of topical Matricaria chamomilla extract activity on linear incisional wound healing in albino rats. Nat Prod Res. 2010; 24(8):697-702.

22. Kurcubic V, Maskovic P, Vujic J, Vranic D, Veskovic-Moracanin S, Okanovic $Đ$ et al. Antioxidant and antimicrobial activity of Kitaibelia vitifolia extract as alternative to the added nitrite in fermented dry sausage. Meat Sci. 2014;97(4):459-67.

23. Oliveira I, Sousa A, Ferreira ICFR, Bento A, Estevinho L, Pereira JA. Total phenols, antioxidant potential and antimicrobial activity of walnut (Juglans regia L.) green husks. Food Chem Toxicol. 2008;46(7):2326-31.

24. Tekwu EM, Pieme AC, Beng VP. Investigations of antimicrobial activity of some Cameroonian medicinal plant extracts against bacteria and yeast with gastrointestinal relevance. J Ethnopharmacol. 2012;142(1):265-73.

25. Manojlovic N, Rankovic B, Kosani M, Vasiljevic P, Stanojkovic T. Chemical composition of three Parmelia lichens and antioxidant, antimicrobial and cytotoxic activities of some their major metabolites. Phytomedicine. 2012;19(13):1166-72.

26. Vieira DRP, Amaral FMM, Maciel MCG, Nascimento FRF, Liberio SA, Rodrigues VP. Plant species used in dental diseases: ethnopharmacology aspects and antimicrobial activity evaluation. J Ethnopharmacol. 2014; 155(3):1441-49.

27. Aleksic V, Knezevic P. Antimicrobial and antioxidative activity of extracts and essential oils of Myrtus communis L. Microbiol Res. 2014;169(4):240-54.

28. Moricz AM, Ott PG, Alberti A, Boszormenyi A, Lemberkovics E, Szoke E et al. Applicability of preparative overpressured layer chromatography and direct bioautography in search of antibacterial chamomile compounds. J AOAC Int. 2013; 96(6):1214-21.

29. Silva NCC, Barbosa L, Seito LN, Fernandes Junior A. Antimicrobial activity and phytochemical analysis of crude extracts and essential oils from medicinal plants. Nat Prod Res. 2012;26(16):1510-14.

30. Carvalho AF, Silva DM, Silva TRC, Scarcelli E, Manhani MR. Avaliação da atividade antibacteriana de extratos etanólico e de ciclohexano a partir das flores de camomila (Matricaria chamomilla L.). Rev Bras Pl Med. 2014;16(3):521-26.

31. Albuquerque ACL, Pereira MSV, Pereira JV, Costa MRM, Pereira LF, Higino JS. Efeito antimicrobiano do extrato da Matricaria recutita Linn. (camomila) sobre microrganismos do biofilme dental. Pesq Bras Odontoped Clin Integr. 2010;10(3):451-55.

32. Agatonovic-Kustrin S, Ortakand DB, Morton DW, Yusof AP. Rapid evaluation and comparison of natural products and antioxidant activity in calendula, feverfew, and German chamomile extracts. J Chromatogr A. 2015;1385:103-10.

33. Capuzzo A, Occhipinti A, Maffei ME. Antioxidant and radical scavenging activities of chamazulene. Nat Prod Res. 2014;28(24):2321-23.

34. Jabri MA, Sani M, Rtibi K, Marzouki L, El-Benna J, Sakly $\mathrm{M}$ et al. Chamomile decoction extract inhibits human neutrophils ROS production and attenuates alcohol-induced haematological parameters changes and erythrocytes oxidative stress in rat. Lipids Health Dis. 2016;15:65.

35. Kolodziejczyk-Czepas J, Bijak M, Saluk J, Ponczek MB, Zbikowska HM, Nowak P et al. Radical scavenging and antioxidant effects of Matricaria chamomilla polyphenolicpolysaccharide conjugates. Int J Biol Macromol. 2015;72:1152-58.

36. Pereira SV, Reis RASP, Garbuio DC, Freitas LAP. Dynamic maceration of Matricaria chamomilla inflorescences: optimal conditions for flavonoids and antioxidant activity. Rev Bras Farmacogn. 2018;28(1):111-17. 
37. Miguel FG, Cavalheiro AH, Spinola NF, Ribeiro DL, Barcelos GR, Antunes LM et al. Validation of a RPHPLC- DAD method for chamomile (Matricaria recutita) preparations and assessment of the marker, apigenin-7-glucoside, safety and anti-inflammatory effect. Evid Based Complement Alternat Med. 2015;2015:828437.

38. Drummond EM, Harbourne N, Marete E, Jacquier JC, O'Riordan D, Gibney ER. An in vivo study examining the antiinflammatory effects of chamomile, meadowsweet, and willow bark in a novel functional beverage. J Diet Suppl. 2013;10(4):370-80.

39. Batista ALA, Lins RDAU, Coelho RS, Barbosa DN, Belem NM, Celestino FJA. Clinical efficacy analysis of the mouth rinsing with pomegranate and chamomile plant extracts in the gingival bleeding reduction. Complement Ther Clin Pract. 2014;20(1): 93-8.

40. Rocha NF, Rios ER, Carvalho AM, Cerqueira GS, Lopes AA, Leal LKAM et al. Anti-nociceptive and anti-inflammatory activities of $(-)$ - $\alpha$-bisabolol in rodents. Naunyn Schmiedebergs Arch Pharmacol. 2011;384(6):525-33.

41. Goes P, Dutra CS, Lisboa MRP, Gondim DV, Leitão R, Brito GAC et al. Clinical efficacy of a $1 \%$ Matricaria chamomile L. mouthwash and $0.12 \%$ chlorhexidine for gingivitis control in patients undergoing orthodontic treatment with fixed appliances. J Oral Sci. 2016;58(4):569-74.

42. Yeung KS, Hernandez M, Mao JJ, Haviland I, Gubili J. Herbal medicine for depression and anxiety: A systematic review with assessment of potential psycho-oncologic relevance. Phytother Res. 2018;32(5):865-91.

43. Chang SM, Chen $\mathrm{CH}$. Effects of an intervention with drinking chamomile tea on sleep quality and depression in sleep disturbed postnatal women: a randomized controlled trial. J Adv Nurs. 2016; 72(2):306-15.

44. Mao JJ, Xie SX, Keefe JR, Soeller I, Li QS, Amsterdam JD. Long-term chamomile (Matricaria chamomilla L.) treatment for generalized anxiety disorder: A randomized clinical trial. Phytomedicine. 2016;23(14):1735-42.

45.Zick SM, Wright BD, Sen A, et al. Preliminary examination of the efficacy and safety of a standardized chamomile extract for chronic primary insomnia: A randomized placebocontrolled pilot study. BMC Complement Altern Med. 2011;11:78.

46. Choi MY, Min ES, Hur MH, Lee MS. Effect of aromatherapy on the anxiety, vital signs, and sleep quality of percutaneous coronary intervention patients in intensivecare units. Evid Based Complement Alternat Med. 2013;2013:381381.
47. Tolouee M, Alinezhad S, Saberi R, Eslamifar A, Zad SJ, Jaimand $\mathrm{K}$ et al. Effect of Matricaria chamomilla L. flower essential oil on the growth and ultrastructure of Aspergillus niger van Tieghem. Int J Food Microbiol. 2010;139(3):127-33. 48. Jamalian A, Shams-Ghahfarokhi M, Jaimand K, Pashootan N, Amani A, Razzaghi-Abyaneh M. Chemical composition and antifungal activity of Matricaria recutita flower essential oil against medically important dermatophytes and soil-borne pathogens. J Mycol Med. 2012;22(4):308-15.

\section{CONFLITO DE INTERESSES}

Os autores declaram não haver conflitos de interesse.

AUTOR PARA CORRESPONDENCIA

José Henrique de Araújo Cruz

henrique_araujo1992@ hotmail.com

Submetido em 26/03/2019

Aceito em 28/08/2019 